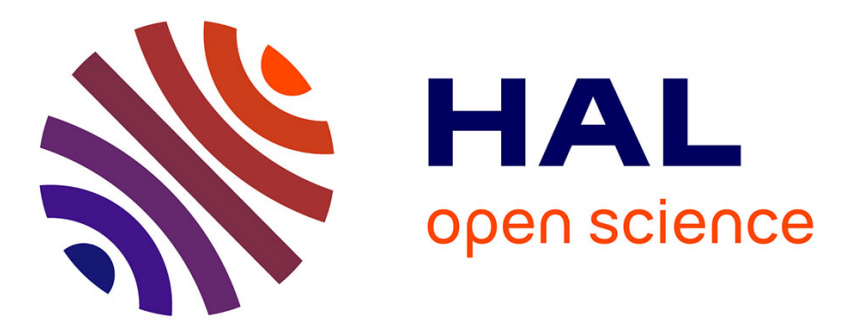

\title{
La femme est le rêve du citadin : chansons populaires de Brazzaville
}

\author{
Augustin-Marie Milandou
}

\section{To cite this version:}

Augustin-Marie Milandou. La femme est le rêve du citadin: chansons populaires de Brazzaville. Journal des anthropologues, 1995, $\mathrm{n}^{\circ}$ 61-62 (L'imaginaire de la ville, sous le direction de Catherine Choron-Baix et Marc Piault), pp.147-153. halshs-00006997

\section{HAL Id: halshs-00006997 https://shs.hal.science/halshs-00006997}

Submitted on 11 May 2008

HAL is a multi-disciplinary open access archive for the deposit and dissemination of scientific research documents, whether they are published or not. The documents may come from teaching and research institutions in France or abroad, or from public or private research centers.
L'archive ouverte pluridisciplinaire HAL, est destinée au dépôt et à la diffusion de documents scientifiques de niveau recherche, publiés ou non, émanant des établissements d'enseignement et de recherche français ou étrangers, des laboratoires publics ou privés. 


\title{
LA FEMME EST LE RÊVE DU CITADIN Chansons populaires de Brazzaville
}

\author{
Augustin-Marie MILANDOU \\ (CNRS, UPR03)
}

De 1989 à 1992, j'ai entrepris une ethnologie de la vie de voisinage dans les quarticrs pauvres de Brazzaville ', capitale du Congo, ma ville natale. Pour être revenu dans cette ville après de longues années de séjour ininterrompu à l'étranger, j'avais été frappé par une réalité qui du temps où j'y étais n'avait jamais interpellé ma curiosité scientifique, tant elle faisait intimement partie de mon quotidien : la musique populaire. J'avais été frappé par son lien avec les milieux dont elle était l'émanation et la profondeur de ce que, sous les thèmes les plus ordinaires, elle disait de la ville. J'en avais fait dans mon enquête une source orale privilégiée. Mais c'était son réalisme qui alors m'intéressait. C'est en la reconsidérant en elle-même que je lui ai trouvé une dimension véritablement imaginaire. La musique populaire dit la ville pour les milieux dont elle est l'émanation mais elle dit aussi ce qu'elle n'est pas ou ce qu'elle devrait être.

\section{La musique populaire}

«Création de blancs, la ville se peuple de noirs », écrivait Jean Dresch dans les années $1940^{2}$. Poste administratif en 1880 ,

\footnotetext{
1 Augustin-Marie MILANDOU, Par delà la vie de voisinage. Le pauvre de Brazzaville et l'existence. Thèse d'anthropologie urbaine. Paris- $V$, juin 1993.

2 J. DRESCH, "Villes congolaises". Rerue de Géographie humaine et d'Ethnologie, III, 1949.
} 
Brazzaville commence à prendre véritablement la forme d'une ville dans les années 1940. Elle est pour les noirs qui s'y installent la ville de toutes les promesses. On travaille toute la journée, mais tous les soirs on se presse dans les bars, pour boire et danser; les samedis et les dimanches sont des jours de réjouissance.

La musique populaire nâ̂t ainsi : du besoin de recréer la chaleur de la vie villageoise. Elle est au départ simplement divertissante. D'ailleurs elle s'applique à reproduire les rythmes des maîtres blancs - la valse, le fox-trot, le tango - et se produit avec des instruments modernes. Antoine Moundaanda avec sa sanza, la guitare traditionnelle, est l'une des rares exceptions ${ }^{3}$. Mais elle va vite devenir la voix privilégiée de l'imaginaire populaire. Car la misère gagne du terrain dans la ville : le chômage et le parasitisme se développent, tandis que les nombreux travailleurs manuels sont sous-payés ${ }^{4}$. La musique populaire est alors la musique de la désillusion, du rêve brisé ; elle dit les infortunes du villageois qui a rêvé de faire fortune en ville. Mais la démographie des quartiers périphériques est à l'époque très particulière. Le sexe masculin est très surreprésenté. Ceux qui se laissent tenter par l'aventurc urbaine dans les villages sont, du fait du poids des traditions, des hommes. Et ils sont jeunes ${ }^{5}$; ils ont besoin de compagnes, pour leur sexualité mais aussi pour échapper à la solitude des retours du travail et des bars.

C'est ainsi que la femme devient objet de compétition, au même titre que l'argent :

$\begin{array}{ll}\begin{array}{l}\text { Makambo mibalé ébomi } \\ \text { Imokili mobimba }\end{array} & \text { Deux choses tuent le monde } \\ \text { Liboso ndé likambo ya falaaka } & \text { La première est l'argent } \\ \text { Ya mibalé likambo ya basi } & \text { La deuxième est la femme }\end{array}$

chantera Kosmos avec les Bantous de la Capitale ${ }^{6}$. La musique po-

\footnotetext{
${ }^{3}$ Sylvain BEMBA, Cinquante ans de musique du Congo-Zaïre, Paris, Présence Africaine, 1984.

4 G. BALANDIER. Sociologie des Brazzavilles noires. Paris, Presses de la Fondation Nationale des Sciences Politiques, 1985, 2ème édition.

5 G. BALANDIER, ibid.

' Kosmos et Les Bantous de la Capitale, années 1960.
} 
pulaire prend la forme du chant d'amour, se fait fidèle interprète de cette situation, c'est-à-dire de ces hommes en quête d'affection et de présence féminine.

Elle est restée cela, même si démographiquement Brazzaville a complètement changé.

On pourrait alors penser qu'elle est devenue un pur produit de l'imagination. Elle ne l'est absolument pas. La musique populaire raconte la ville au travers de l'amour de la femme.

\section{La ville métaphore}

L'amour dans cette musique est très souvent inquiet, malheureux, trahi :

Chérie tika ngai na kufa Bongo sima o boya ngai ${ }^{\text {? }}$ Na yébi bolingo ébandaka Ika lokola sukali é Ékoma na kati ngayi lokola Na suka bololo lokala Inivaquine $^{8}$
Chérie, attends que je meurs Et après tu iras avec un autre Au commencement l'amour

[est sucré

Aigre au milieu

Amer comme la nivaquine [à la fin

L'amour est à l'image de toutes les relations interpersonnelles dans la ville. Les milieux populaires appellent péjorativement cellesci bikamarade ya mboka mundélé (relations du village du blanc). Il ne faut pas voir là une simple figure de style mais une façon de caractériser une manière d'être ensemble. Les bikamarade ya mboka mundélé sont sympathie et précarité. Le citadin est toujours en mouvement.

Le thème de la trahison est omniprésent dans les chansons d'amour. Mais peut-être parce qu'elles sont pour l'essentiel l'oeuvre des hommes, elles présentent davantage la femme comme coupable.

Celle-ci est un sujet insaisissable, qui ne se lasse pas de se laisser séduire. La femme est toujours dans cette chanson une beauté rare - on l'aime pour cette qualité - et désirée de tous les hommes de

\footnotetext{
${ }^{7}$ Djo Poster et Le Grand Zaiko, années 1984-1985.

${ }^{8}$ Lutumba et l'O.K. Jazz, années 1980.
} 
la ville ; elle est toujours objet de compétition :

Mwana mwasi oyo

$O$ whta wapi?

$O$ zali kitoko nanu

Ina mona té !"

Bolingo!

A zali mwasi a vandaka

Ba zali kowéla yé lokola

Ina Lingwala

[Coupe du Monde ! ${ }^{10}$
Jeune fille

D'où viens-tu?

Tu es belle comme je n'en

[ai jamais vue!

Amour !

C'est une fille qui habite

On se la dispute comme

[Lingwala

[la Coupe du Monde !

Tous les prétendants n'ont pas cependant le même poids dans la compétition. Le plus redoutable de tous est le moto ya mbongo, l'homme d'argent, le nanti :

O profité po to swanaki

Ngai na yo Youyou Moké

O kéyi kolingo mwana ya

Imoto ya mbongo

Ngai ko mwana ya mobola

To mékana ndéngé nini ?

Nzongéla ngai mwana mboka

Ngai na bébi awa

Sentiment motéma na ngai

É ké mosika mama

[nyonso Je ne cesse de penser

Kala tozalaka na yo boyé

Kiyédi na ngai

Sûrement ba tambwisi

Na nkasa ya bleue ${ }^{\text {II }}$ lyo moto
Tu as profité d'une dispute

Petite Youyou

Pour aller aimer l'enfant

Moi, l'enfant du pauvre

Comment rivaliser avec lui ?

Reviens, reviens

Je suis devenue une loque

On n'était pas comme ça

Kiyédi, ma chérie

C'est sûr

On t'a tourné la tête

Avec des billets

La chanson d'amour est la plupart du temps une complainte du pauvre (mobola) éconduit au profit du nanti. Elle est hantée par l'argent (mbongo). On dit de la femme qu'elle est comme le poisson, qui va toujours du côté où il y a de l'eau.

Mais le pauvre ne désespère pas pour autant de l'arracher au

"Mimi Kazidonla, Eddy'son, années 1980.

11) Ndombé et l'O.K. Jazz, années 1980.

11 Dino Vagu et L'Afrisa, années 1980. 
nanti. A l'argent il oppose l'incommensurable grandeur de l'amour. L'amour, dit la chanson populaire, est mystérieux, divin, vital. Le chansonnier menace sa belle de se suicider si cllc ne répond pas positivement à son amour :

Bolingo ébanda

Na Adamu na Eva ${ }^{12}$

Na komi na point carré

N'ésika ngai na mélaka
L'amour a commencé

Avec Adam et Eve

Je suis arrivé au point carré

Au point où je bois

[du poison

L'amour est ce qu'il y a de plus humain ; il est don de soi ; il ne peut être monnayé, marchandé, acheté :

Bolingo ya mbongo

E ko wumékala té

Oyo ya motéma suka sé
L'amour d'argent ne dure

[jamais

Celui du coeur dure

[jusqu'à la mort

Ce discours de dévalorisation de l'argent participe de toute une philosophie. La philosophie populaire valorise le muntu (l'être humain). L'être humain est pour elle la richesse suprême, qu'il faut préférer à toute autre chose. L'argent est éloko (rien) parce qu'il ne peut acheter l'amour, l'amitié, la fraternité, la paix, le bonheur, la mort ; parce qu' «il n'a jamais soulevé personne de son lit pour le transporter à l'hôpital ${ }^{15}$.

La dénégation du pouvoir de l'argent est en vérité purement imaginaire. L'argent est l'obsession du Brazzavillois. Pamélo Monk'a, une des grandes figures de la musique populaire, chante cette obsession avec une belle formule dans une composition à grand succès des années 1980 .

Si matili ya insi

Vandiki mbongo

Kasi baatu zégaka matiti

Inyonso
Si les feuilles étaient

Les hommes les auraient

[l'argent

[coupées toutes

\footnotetext{
12 Tabu Ley et l'African Fiesta, années 1968-1970.

${ }^{13}$ Lutumba et Le T.P. O.K. Jazz, années 1980.

${ }^{14}$ Grand Kallé, années 1960.

15 Traduction d'une expression populaire en usage à Brazzaville.
} 
C'est si vrai que l'argent est représenté comme une énigme, quelque chose aux origines mystérieuses :
Mbongo é mbongo
L'argent, l'argent
On'uta wapi é mbongo?
D'où viens-tu?
Mama na yo wapio
Qui est ta mère?

Imbongo? ${ }^{16}$

L'argent occupe l'esprit du citadin du matin jusqu'au soir. Il faut le gagner, aller le chercher là où il est. Mosala zoba ézali té (il n'y a pas de sots métiers), l'argent est le seul critère décisif et il n'a pas d'odeur (mbongo ka zéna n'suunga ko).

La dévalorisation imaginaire de l'argent n'est en vérité que l'expression du dépit à l'égard de la ville. La ville est synonyme d'argent et inversement l'argent signifie la ville. Les milieux populaires - pauvres devrait-on dire - disent de la ville : Oyo mboka té! (Ce n'est pas un lieu où vivre!). Elle n'est pas pour eux mboka moyindu, village du noir, village paisible, moral, égalitaire, solidaire; elle est mboka mundélé, village du blanc. La ville est une immense cité du mal (mokili ya masumu). Une cité à livrer à la géhenne, renchérit le chansonnier :

Mokili oyo

Ébanda mokolo ninio?

Mokili oyo

É kufa mokolo nini o ? ${ }^{17}$
Ce monde

Quand est-il né?

Ce monde

Quand mourra-t-il ?

Comme la dévalorisation de l'argent, dont elle procède, celle de la ville est simulée et très ambivalente. Le Brazzavillois présente sa ville aux campagnards comme un immense lieu de plaisir. D'elle il dit toujours : Béya biséngo (Brazza-la-joie).

Kilumbu ya ntété ti ya sabaala Béto nyonso na bisalu Kasi ntaangu ké kwiza Ba ngaanda nyonso mé fuluka Lunzingu Iwa muntu kaka na [koopo na Brazza ${ }^{18}$
Du lundi au samedi

Tous sommes au travail Mais quand vient la nuit Tous les bars s'emplissent A Brazzaville la vie c'est [le vin

${ }^{16}$ Lutumba et l'O.K. Jazz, années 1980.

17 Pamélo Mounk'a, années 1980. 
Les milieux populaires ne rêvent pas de voir leur ville périr sous des flammes. Ils rêvent d'une ville autre, d'une ville moins inégalitaire, plus humaine, à laquelle ils s'attachent comme le chansonnier à sa belle :

Yangai y

Sé liwa mama ${ }^{19}$

Toi et moi serons unis

Jusqu'à la mort

18 Ange Lineaud, 1985.

${ }^{19}$ Soko Vangu et Les Bella-Bella, années 1970. 